\title{
Co-Design and Control of a Magnetic Microactuator for Freely Moving Platforms
}

\author{
Michael Olbrich ${ }^{1, t, *}$, Arwed Schütz ${ }^{2}$, Koustav Kanjilal ${ }^{3}$, Tamara Bechtold ${ }^{2}$, Ulrike Wallrabe ${ }^{3}$ \\ and Christoph Ament ${ }^{1}$ \\ 1 University of Augsburg, Chair of Control Engineering; christoph.ament@uni-a.de \\ 2 Jade University of Applied Sciences, Research Group for Modelling and Simulation of Mechatronic Systems; \\ arwed.schuetz@jade-hs.de, tamara.bechtold@jade-hs.de \\ 3 University of Freiburg, Department of Microsystems Engineering; koustav.kanjilal@imtek.uni-freiburg.de, \\ wallrabe@imtek.uni-freiburg.de \\ * Correspondence: michael.olbrich@uni-a.de \\ † Current address: Eichleitnerstraße 30, D-86159 Augsburg, Germany
}

\begin{abstract}
A current goal for microactuators is to extend their usually small working ranges, that typically result from mechanical connections and restoring forces imposed by cantilevers. In order to overcome this, we present a bistable levitation setup to realise free vertical motion of a magnetic proof mass. By superimposing permanent magnetic fields, we imprint two equilibrium positions, namely on the ground plate and levitating at a predefined height. Energy-efficient switching between both resting positions is achieved by cooperation of a piezoelectric stack actuator, initially accelerating the proof mass, and subsequent electromagnetic control. A trade-off between robust equilibrium positions and energy-efficient transitions is found by simultaneously optimising the controller and design parameters in a co-design. A flatness-based controller is then proposed for tracking the obtained trajectories. Simulation results demonstrate the effectiveness of the combined optimisation.
\end{abstract}

Keywords: cooperative microactuators; multistability; co-design; control

\section{Introduction}

Microsystem technology has become an increasingly important research field over the past 40 years. Besides microelectronic circuits and miniaturised sensors, microactuators belong to an indispensable sub-branch with applications, e.g., in automotive industry, medicine, and energy harvesting fields. The versatile applicability originates from their ability to produce precise mechanical movements on a micrometer scale. However, the range of motion is in general limited by their design. In order to overcome this, various approaches have been proposed. Breguet et al. presented a piezoelectric stick and slip actuator that achieves accurate linear movements up to several centimetres [1]. In the study of Mita et al., an internally suspended mass is electrostatically driven, such that the actuator is moved by the impact between the mass and a stopper [2]. Since friction is a key element in such concepts, a modelling approach for stick and slip actuators is given by Edeler et al. [3]. Large working ranges can also be achieved by electromagnetic levitation. This principle is used for the design of a linear microactuator, in which a platform is levitated and able to move in longitudinal direction $[4,5]$. Stability of levitation by inductive suspensions is proven by Poletkin et al. [6]. In general, the presented approaches suffer from various drawbacks, e.g., permanent energy consumption for creating any stable equilibrium position in case of electromagnetic levitation or the high dependence on friction. The latter corresponds to a loss of energy due to the undesirable conversion from kinetic energy into dissipated heat. Moreover, the performance heavily depends on the surface condition. In case of 
predefined resting positions and unidirectional motion, pseudo-levitation as in magnetic bearings [7] can be applied by using permanent magnetic fields.

Besides a suitable choice of the working principle, a controlled motion is essential for precision and effectiveness. For small displacements, a common choice is to linearise the system at an operating point to describe the local behaviour. In this case, linear control strategies can give satisfactory results [8]. For enlarged working ranges, however, nonlinearities can in general not be neglected. Advanced control methods such as flatness-based control $[9,10]$ or backstepping [11] can be used to obtain precise motion. The overall efficiency of the controlled state trajectory, however, can strongly depend on the chosen actuator design. For an optimal outcome, both the design and controller should therefore be optimised simultaneously in a co-design, as shown in [12] for an electrostatically driven actuator.

In this work, we introduce a cooperative, bistable microactuator setup, using both magnetic and piezoelectric actuation. The goal is to achieve stable equilibrium positions without the need for permanent energy input. The transition between the two imprinted resting positions is accomplished by an efficient control strategy that exploits the cooperation of the two single actuators, namely a piezoelectric stack actuator and an electromagnetic solenoid. To ensure an optimal trade-off between low energy consumption and the robustness of the resting positions, both the actuator design and controller are optimised simultaneously in a co-design.

\section{Methods}

We consider the cooperative microactuator system shown in Figure 1, where a magnetic proof mass within a glass tube can move between two equilibrium positions, namely resting on top of a piezoelectric stack actuator and levitating at a predefined upper position. This corresponds to the design presented by Schütz et al. [13], but with the extension of a second permanent magnet and the stack actuator. Starting at the lower equilibrium, the inverse piezoelectric effect is used to apply an impulse-like acceleration, i.e., a kick, to the proof mass. This is followed by a controlled electromagnetic catch at an upper equilibrium position, where the mass is stably suspended by the permanent magnetic field.

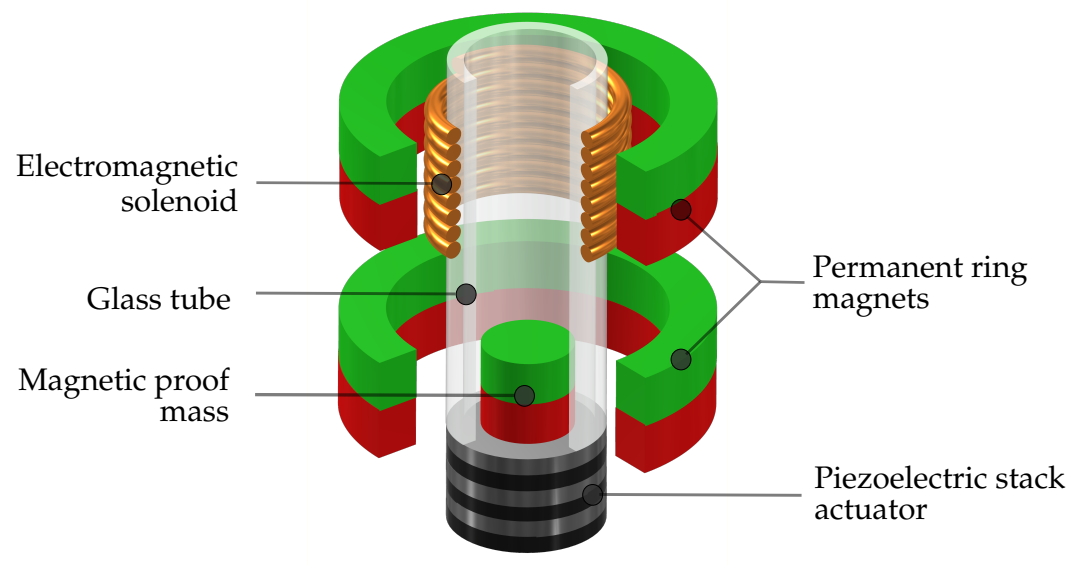

Figure 1. The schematic of the magnetic microactuator is shown. It consists of a movable proof mass within a guiding glass tube, a piezoelectric stack actuator (kick actuator), permanent magnets and an electromagnet (catch actuator).

The objective is to achieve a robust, bistable characteristic without the need for a permanent energy input, as well as a fast and efficient transition between the resting positions. Therefore, a parameterised system model is derived and then optimised simultaneously with the controller in a co-design. 


\subsection{Modelling}

The actuator consists of three coupled dynamical systems, namely a solenoid, a stack actuator and a movable mass. The current $i(t)$ of the electromagnet is given as

$$
L \frac{\mathrm{d}}{\mathrm{d} t} i(t)=u_{\mathrm{in}}(t)-R i(t),
$$

where $R$ denotes the wire resistance, $L$ the inductance, and $u_{\text {in }}(t)$ the input voltage. For the piezoelectric actuator, a linear second order approximation is used [14]. Taking into account the effectively moved mass $M$ of the piezo actuator, which is assumed to be a third of its overall mass, the deformation $d(t)$ can be described by

$$
M \ddot{d}(t)=-M g-c_{\mathrm{A}} \dot{d}(t)-k_{\mathrm{A}} d(t)-F_{\mathrm{c}}(t)+F_{\mathrm{u}}(t),
$$

where $g$ is the gravitational acceleration, $c_{\mathrm{A}}$ the damping and $k_{\mathrm{A}}$ the stiffness coefficients of the actuator, respectively. The forces $F_{\mathrm{c}}(t)$ and $F_{\mathrm{u}}(t)$ correspond to the contact force between the piezo actuator and the proof mass and the inverse piezoelectric effect by applying a voltage $u_{\mathrm{A}}(t)$. Similar to [15], we approximate the relation between the force and input voltage by a linear equation, hence

$$
F_{\mathrm{u}}(t)=\frac{F_{\max }}{U_{\max }} u_{\mathrm{A}}(t) .
$$

In (3), $F_{\max }$ and $U_{\max }$ are the maximum achievable force and its corresponding input voltage, respectively. Although the dynamics are nonlinear, we assume the model to be sufficiently representative for the short time of contact with the proof mass. Finally, the vertical motion $z(t)$ of the proof mass is determined by the sum of forces

$$
m \ddot{z}=F_{\mathrm{pm}}(z)+F_{\mathrm{em}}(z, i)+F_{\mathrm{c}}(z, \dot{z}, d, \dot{d})-c_{\mathrm{f}} \dot{z}|\dot{z}|-m g .
$$

Note that the time dependencies have been neglected for readability. The parameters $c_{\mathrm{f}}$ and $m$ denote the air friction coefficient and the mass of the proof mass. Moreover, the magnetic interaction is described by the permanent and electromagnetic forces $F_{\mathrm{pm}}(z), F_{\mathrm{em}}(z, i)$, where the former corresponds to a superposition of the single forces $F_{\mathrm{pm}, j}(z), j=1,2$. We assume fully magnetised, hard magnetic materials with remanence values $B_{\mathrm{r}, \mathrm{p}}, B_{\mathrm{r}, \mathrm{pm}, j}$ for the proof mass and ring magnets. Thus, we can use the proportional relations $\left(B_{\mathrm{r}, \mathrm{p}} B_{\mathrm{r}, \mathrm{pm}, j}\right) \sim F_{\mathrm{pm}, j}(z)$ and $\left(B_{\mathrm{r}, \mathrm{p}} i\right) \sim F_{\mathrm{em}}(z, i)[16,17]$. Due to the linearity, we can now describe the magnetic forces by introducing $z$-dependent shape functions $f_{\mathrm{pm}, j}(z), f_{\mathrm{em}}(z)$ as

$$
\begin{aligned}
& F_{\mathrm{pm}, j}(z)=B_{\mathrm{r}, \mathrm{p}} B_{\mathrm{r}, \mathrm{pm}, j} f_{\mathrm{pm}, j}(z), \\
& F_{\mathrm{em}}(z, i)=B_{\mathrm{r}, \mathrm{p}} i f_{\mathrm{em}}(z) .
\end{aligned}
$$

Instead of an analytical expression, these forces are computed numerically with ANSYS ${ }^{\circledR}$ Maxwell [18] for a grid of system states and parameters and are implemented as a spline-interpolated lookup table. It is noteworthy that we can inversely compute the shape functions from $(5,6)$, given the numerical simulation data of the forces with known remanence values and currents. In case the permanent and electromagnet centre points are given by $z_{\mathrm{pm}, j}$ and $z_{\mathrm{em}}$, the force can easily be obtained by shifting the shape function.

For the computation of the contact force $F_{\mathrm{c}}(z, \dot{z}, d, \dot{d})$ between the piezoelectric actuator and the movable mass a viscoelastic approximation adapted from the method of power-based restriction functions [19] is applied. Thus, by allowing a small overlap $s_{\perp}(t)=z(t)-d(t)$ between both objects, the counteracting force is proportional to an activation function $\mathcal{R}_{\mathrm{a}}\left(s_{\perp}\right)$, which corresponds to a virtual, nonlinear spring. In contrast to the latter reference, we use the piecewise function 


$$
\mathcal{R}_{\mathrm{a}}\left(s_{\perp}\right)= \begin{cases}0, & \text { if } s_{\perp} \geq 0 \\ -k s_{\perp}+\tau k\left(\exp \left(\frac{s_{\perp}}{\tau}\right)-1\right), & \text { otherwise }\end{cases}
$$

For further details, the reader is referred to the corresponding work of Specker et al. [19].

\subsection{Trajectory Planning and Control}

The derived model is now used to find an efficient control strategy. We first consider both actuation principles separately and then give an outline of the overall approach.

\subsubsection{Electromagnetic actuation}

Let us first highlight two relevant system properties of the electromagnetic actuation. On the one hand, the electromagnetic force is zero whenever the position of the proof mass coincides with the centre point of the solenoid, hence $F_{\mathrm{em}}\left(z_{\mathrm{em}}, i\right)=0, \forall i \in \mathbb{R}$. On the other hand, the model is flat with respect to the output $z(t)$, allowing us to express the system states and input exactly by only the output and its derivatives [20]. Consider the assumption that $z(t) \neq z_{\mathrm{em}}, \forall t$.

We then predetermine a sufficiently differentiable output trajectory $z_{\text {ref }}(t)$. By definition of the flatness property, there exists a reversible state transformation between $x=[z, \dot{z}, i]$ and $\xi=[z, \dot{z}, \ddot{z}]$. This can be used to inversely compute the reference input sequence

$$
u_{\text {in,ref }}(t)=f^{-1}\left(z_{\text {ref }}, \dot{z}_{\text {ref }}, \ddot{z}_{\text {ref }}, \dddot{z}_{\text {ref }}\right) \text {, }
$$

where $f^{-1}(\cdot)$ corresponds to the inverse relation between the output, its derivatives and the solenoid voltage after the state transformation, and can be found by reformulating $(1,4)$. That is, by feedforward application of $u_{\mathrm{in}, \text { ref }}(t)$ and in the absence of disturbances, we achieve the desired reference motion $z_{\text {ref }}(t)$ without error, provided that the initial conditions are satisfied.

The reference input is now superimposed by an additional feedback controller for disturbance compensation. Here, we make use of the linearisability of the system via a feedback term [21]. By using the state transformation, we obtain the linear system

$$
\dot{\xi}=\left[\begin{array}{lll}
0 & 1 & 0 \\
0 & 0 & 1 \\
0 & 0 & 0
\end{array}\right]+\left[\begin{array}{l}
0 \\
0 \\
1
\end{array}\right] v,
$$

with a virtual input $v(t)$. Since $v=\dddot{z}(t)$, we can insert it into (8) and obtain an expression for $u_{\text {in }}(t)$ in dependence of $v(t)$. Thus, the task is reduced to controlling the linear system. We propose a Two-degree-of-freedom controller design, consisting of the feedforward term $v_{\text {ref }}(t)=\dddot{z}_{\text {ref }}(t)$, and a feedback control law $\triangle v(t)=-\boldsymbol{K} \boldsymbol{e}(t)$, resulting in $v(t)=v_{\text {ref }}(t)+\Delta v(t)$. Here, $\boldsymbol{e}(t)=\boldsymbol{\xi}_{\text {ref }}(t)-\boldsymbol{\xi}(t)$ is the state error and the stabilising controller gain matrix $K$ is found in terms of a linear quadratic regulator (LQR).

For the feedfoward term, a suitable reference needs to be computed. The goal is to find a twice differentiable motion $z_{\text {ref }}(t)$ starting at initial state $\xi_{0}$ and terminating at $\xi_{\mathrm{f}}$ after time $T_{\mathrm{f}}$. We achieve this by defining $\dddot{z}_{\text {ref }}(t)$ and subsequently integrating it. Here, we choose $\dddot{z}_{\text {ref }}(t)$ to be piecewise constant with $n \geq 3$ equidistant time steps $T$, i.e.,

$$
\dddot{z}_{\text {ref }}(t)=u_{i}, t \in[T i, T(i+1)], \forall i=0, \ldots, n-1 .
$$

These trajectory parameters $u_{i}$ can be chosen arbitrarily. Only the last three parameters $u_{n-j}, j=1,2,3$ have to be computed in a way that the three final state conditions are met. 


\subsubsection{Piezoelectric actuation}

So far, we only focused on the electromagnetic actuation. Since there is a large distance between the lower equilibrium and the solenoid, initially accelerating the proof mass requires high electrical currents. A more suitable approach is to transfer the relevant force directly by a piezoelectric kick. Due to the short interaction time between the mass and the piezo actuator, we only use feedforward control in terms of a voltage spike. Here, the applied voltage

$$
u_{\mathrm{A}}(t)=\frac{u_{\mathrm{p}}}{0.53}\left(\exp \left(\frac{-t}{\tau_{2}}\right)-\exp \left(\frac{-t}{\tau_{1}}\right)\right)
$$

with $\tau_{1}=1 \mathrm{~ms}, \tau_{2}=5 \mathrm{~ms}$ and the peak voltage $u_{\mathrm{p}} \leq U_{\max }$ is used as an approximation of the achievable voltage. In summary, the cooperative actuation starts with a kick, and the electromagnetically controlled catch is switched on after time $T_{\text {kick }}$. The subsequently followed trajectory is precomputed such that it ends in the upper equilibrium position with zero velocity and acceleration. The permanent magnets then stably keep the proof mass in place. The reverse procedure is used for the direction to the lower resting position. However, only the electromagnet can then be used.

\subsection{Co-Design}

For a fixed system, efficient trajectories can now be obtained by an optimisation over the trajectory parameters $u_{i}$. However, the system design influences the overall optimality, which is indicated by the permanent magnetic force acting on the proof mass, as shown in Figure 2 for two different designs.

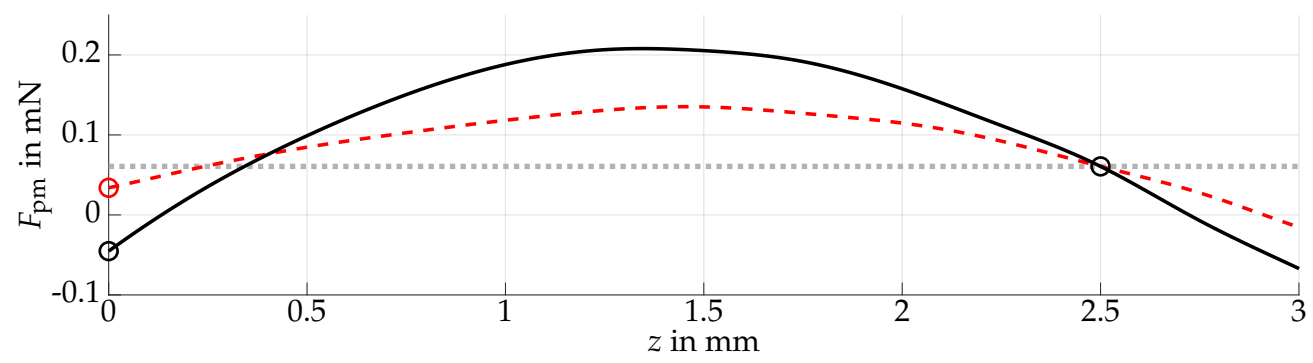

Figure 2. Stationary magnetic force characteristics depending on the proof mass position for two different magnet setups. Both the strong magnetic field (solid, black) and the weak field (dashed, red) have stable equilibrium positions at $z=0$ and $z=2.5 \mathrm{~mm}$. The dotted, grey line corresponds to the magnitude of the gravitational force.

The equilibrium positions correspond to the intersection of the mirrored gravitational force $F_{\mathrm{g}}$ with the magnetic and contact force characteristics. These are locally stable, if additionally, the force gradient is negative. Due to the counterforce of the stack actuator at the zero position, the stability criterion at this point is simplified, i.e., the point is stable if the magnetic force is lower than $F_{\mathrm{g}}$. This corresponds to the mass being pushed against the piezo actuator. For both setups shown in Figure 2, the resting positions are located at $z=0 \mathrm{~mm}$ and $z=2.5 \mathrm{~mm}$, and their robustness depends on the force steepness around that point. A steep force is able to counteract small deviations, pushing the proof mass back into the resting position. In case of the flat curve, the positive magnetic force compensates most of the gravitational force at the lower position. Thus, a small positive force disturbance is sufficient for the proof mass to inadvertently leave its equilibrium. Therefore, robust equilibria are preferred. However, the energy effort for the transition between these points increases with robustness, since larger counterforces have to be overcome. Thus, a trade-off has to be found. These and similar contradictory goals can be handled by simultaneously optimising the system and the controller in a co-design. 
Consider the individual design and controller cost functions $J_{\mathrm{d}}\left(p_{\mathrm{d}}\right), J_{\mathrm{t}}\left(p_{\mathrm{d}}\right)$, depending on the respective parameter vectors $p_{\mathrm{d}}, p_{\mathrm{t}}$, where the indices $\mathrm{d}$ and $\mathrm{t}$ denote the design and the trajectory. See Table 2 for an overview of the parameters. Since the controller costs are additionally influenced by the design parameters, minimising both cost functions individually can lead to suboptimal overall behaviour. Instead, we define a combined cost function $J(p)$ with the extended parameter vector $p=\left[p_{\mathrm{d}}, p_{\mathrm{t}}\right]$, and simultaneously optimise both the design and controller. With the respective weightings $w_{\mathrm{d}}$ and $w_{\mathrm{t}}$ the optimisation can mathematically be expressed as

$$
\begin{aligned}
& \min _{\boldsymbol{p}}\left(w_{\mathrm{d}} J_{\mathrm{d}}(\boldsymbol{p})+w_{\mathrm{t}} J_{\mathrm{t}}(\boldsymbol{p})\right) \\
& \text { s.t. } \boldsymbol{p} \in \mathcal{P},
\end{aligned}
$$

where $\mathcal{P}$ denotes the constrained set of allowed parameters. The design costs evaluate stability and robustness, solely depending on the permanent magnetic field. Here, we penalise the deviation from reference forces $F_{\text {ref,low }}, F_{\text {ref,up }}$ and the upper equilibrium gradient $\nabla F_{\text {ref,up }}$, resulting in

$$
\begin{aligned}
J_{\mathrm{d}}=w_{\mathrm{d}, 1}\left(F_{\mathrm{pm}}\left(z_{\text {eq,low }}\right)-F_{\text {ref,low }}\right)^{2} & +w_{\mathrm{d}, 2}\left(F_{\mathrm{pm}}\left(z_{\text {eq }, \text { up }}\right)-F_{\mathrm{g}}\right)^{2} \\
& +w_{\mathrm{d}, 3}\left(\nabla F_{\mathrm{pm}}\left(z_{\text {eq }, \text { up }}\right)-\nabla F_{\text {ref,up }}\right)^{2} .
\end{aligned}
$$

For given component dimensions, $F_{\mathrm{pm}}$ depends on the remanence of both the proof mass and the magnetic rings, as well as the positions $z_{\mathrm{pm}, 1}, z_{\mathrm{pm}, 2}$. For simplicity of the optimisation, we used a continuous set of remanence values, assuming that the final result can approximately be achieved by custom composite magnets of individual shape and dimension. The design can be then optimised over the parameters $B_{\mathrm{r}, j}, z_{\mathrm{pm}, j}, j=1,2$ and $B_{\mathrm{r}, p}$. Concerning the trajectory efficiency, the quadratic cost function

$$
J_{\mathrm{t}}=\sum_{k=1}^{N} w_{\mathrm{t}, 1}\left(z_{k}-z_{\mathrm{eq}, k}\right)^{2}+w_{\mathrm{t}, 2} \dot{z}_{k}^{2}+w_{\mathrm{t}, 3} i_{k}^{2}+w_{\mathrm{t}, 4} u_{\mathrm{in}, k}^{2}
$$

is used, which penalises the deviation from the current reference equilibrium $z_{\mathrm{eq}} \in\left\{z_{\mathrm{eq}, \mathrm{low}}, z_{\mathrm{eq}, \mathrm{up}}\right\}$, high velocities, solenoid currents and the input effort at sampled time steps $t=k T_{\mathrm{s}}$ with step width $T_{\mathrm{s}}$. The costs are directly influenced by the parameter vector $p_{\mathrm{t}}$, containing the values $u_{i}$, the maximum transition time $T_{\mathrm{f}}$, the piezo voltage peak $u_{\mathrm{p}}$, and the controller switch on time $T_{\text {kick }}$. Additionally, the efficiency indirectly depends on the design parameters of the magnetic field, as well as the solenoid center position $z_{\mathrm{em}}$. The overall cost function can then be minimised by a suitable constrained optimisation algorithm.

\section{Results}

The co-design framework is implemented in $\operatorname{MATLAB}^{\circledR}$ and the dynamics $(1,2,4)$ are integrated numerically using the stiff ode23t solver. The optimisation problem is non-convex and partially discontinuous. The latter results from the piezoelectric kick and the stiffness of the contact model. Therefore, a genetic algorithm of the global optimisation toolbox [22] is used. See Table 1 for the fixed component and control parameters, and Table 2 for an overview of the optimisation parameters and their linear bounds. The existence of both equilibrium positions is ensured by shifting the magnetic field to match the upper resting position, and using a nonlinear constraint $F_{\mathrm{pm}}(0) \leq 0.5 F_{\mathrm{g}}$ for the lower position. For the following study, we used the weighting coefficients given in Table 3 . 
Table 1. Fixed design and control parameters that are used in simulation

\begin{tabular}{ll|ll}
\hline Description & Value & Description & Value \\
\hline Inner radius (ring magnets) & $4.5 \mathrm{~mm}$ & $U_{\max }$ (piezo) & $100 \mathrm{~V}$ \\
Outer radius (ring magnets) & $5.0 \mathrm{~mm}$ & Stiffness (piezo) & $1.0909 \mathrm{kNm}^{-1}$ \\
Height (ring magnets) & $1.5 \mathrm{~mm}$ & Surface (piezo) & $9 \mathrm{~mm}^{2}$ \\
Inner radius (solenoid) & $0.8 \mathrm{~mm}$ & Height (piezo) & $21 \mathrm{~mm}$ \\
Outer radius (solenoid) & $2.5 \mathrm{~mm}$ & Mass (piezo) & $1.8 \mathrm{~g}$ \\
Height (solenoid) & $1.5 \mathrm{~mm}$ & Diameter (proof mass) & $1.0 \mathrm{~mm}$ \\
Wire diameter (solenoid) & $25 \mu \mathrm{m}$ & Height (proof mass) & $1.0 \mathrm{~mm}$ \\
Specific resistance (solenoid) & $1.8 \times 10^{-8} \Omega \mathrm{m}$ & Density (proof mass) & $7874 \mathrm{kgm}^{-3}$ \\
Number of coils (solenoid) & 4000 & State penalty $Q$ (LQR) & $\operatorname{diag}([5 \mathrm{e} 12,2 \mathrm{e} 9,2 \mathrm{e} 3])$ \\
$F_{\max }$ (piezo) & $360 \mathrm{~N}$ & Input penalty $R(\mathrm{LQR})$ & 1 \\
\hline
\end{tabular}

Table 2. Optimisation variables $p_{\mathrm{d}}$ and $p_{\mathrm{t}}$ with corresponding linear bounds used in the co-design

\begin{tabular}{lllll}
\hline Variable & Description & Lower bound & Upper bound & Optimised value \\
\hline$B_{\mathrm{r}, \mathrm{pm}, 1}$ & Ring magnet remanence & $0.1 \mathrm{~T}$ & $1 \mathrm{~T}$ & $0.2433 \mathrm{~T}$ \\
$B_{\mathrm{r}, \mathrm{pm}, 2}$ & Ring magnet remanence & $-1 \mathrm{~T}$ & $1 \mathrm{~T}$ & $0.1425 \mathrm{~T}$ \\
$B_{\mathrm{r}, \mathrm{p}}$ & Proof mass remanence & $0.01 \mathrm{~T}$ & $1 \mathrm{~T}$ & $0.9977 \mathrm{~T}$ \\
$z_{\mathrm{pm}, 1}$ & Position of ring magnet 1 & $1 \mathrm{~mm}$ & $10 \mathrm{~mm}$ & $2.773 \mathrm{~mm}$ \\
$z_{\mathrm{pm}, 2}$ & Position of ring magnet 2 & $-7.5 \mathrm{~mm}$ & $1 \mathrm{~mm}$ & $-5.329 \mathrm{~mm}$ \\
$z_{\mathrm{em}}$ & Position of solenoid & $2.8 \mathrm{~mm}$ & - & $3.087 \mathrm{~mm}$ \\
\hline$u_{\mathrm{p}}$ & Piezoactuator voltage peak & $0 \mathrm{~V}$ & $U_{\mathrm{max}}$ & $65.78 \mathrm{~V}$ \\
$u_{i}$ & Third motion derivative & - & - & - \\
$T_{\mathrm{kick}}$ & Controller switch on time & $0 \mathrm{~s}$ & $0.02 \mathrm{~s}$ & $3.71 \mathrm{~ms}$ \\
$T_{\mathrm{f}}$ & Maximum transient time & $1.1 T_{\text {kick }}$ & $T_{\text {sim }}$ & $97.12 \mathrm{~ms}$ \\
\hline
\end{tabular}

Table 3. Weighting coefficients of cost functions

\begin{tabular}{ll|ll}
\hline Parameter & Value & Parameter & Value \\
\hline$w_{\mathrm{d}}$ & 8 & $w_{\mathrm{t}, 1}$ & $2.22 \mathrm{e} 04$ \\
$w_{\mathrm{d}, 1}$ & 28 & $w_{\mathrm{t}, 2}$ & 0 \\
$w_{\mathrm{d}, 2}$ & $w_{\mathrm{t}, 3}$ & 0.25 \\
$w_{\mathrm{d}, 3}$ & $55 \mathrm{e} 06$ & $w_{\mathrm{t}, 4}$ & $2.22 \mathrm{e} 07$ \\
$w_{\mathrm{t}}$ & $70 \mathrm{e} 07$ & & \\
\hline
\end{tabular}

The trajectory to be optimised consists of a motion to the upper equilibrium $z_{\text {eq,up }}=2.5 \mathrm{~mm}$ within $T_{\text {sim }}=0.15 \mathrm{~s}$, and back to the lower position within the same time span. The actual transient time $T_{\mathrm{f}}$ is divided into $n=9$ equidistant intervals $T$, to which an individual, optimised motion parameter $u_{i}$ is assigned. The result is shown in Figure 3.

As it can be seen in Figure 3a, fast trajectories in both, upwards and downwards direction are achieved with small overshoot and a transition time of $T_{\mathrm{f}}=97.1 \mathrm{~ms}$. The optimised variables are given in Table 2. The bistability property is verified by both, zero inputs at the equilibrium positions and magnetic field, which almost matches the reference points. In Figure 3a, the advantage of using the cooperation between the piezoelectric kick and the catch is clearly illustrated. Due to the kick, the proof mass is accelerated to a velocity of $0.1 \mathrm{~ms}^{-1}$, largely reducing the necessary voltage input. Without the piezoelectric input, the optimal trajectory requires an initial voltage of $38 \mathrm{~V}$ for achieving the same transient time. This is also reflected by the trajectory costs $J_{t}$, which are 0.284 for the cooperative solution and 0.352 for the simulation without the kick.

Given the optimised reference, we now study the trajectory following controller in simulation. To achieve this, the reference is used as a lookup-table for the feedforward term. In order to assess the disturbance compensation ability, model uncertainty is implemented, i.e., the magnetic field for the 


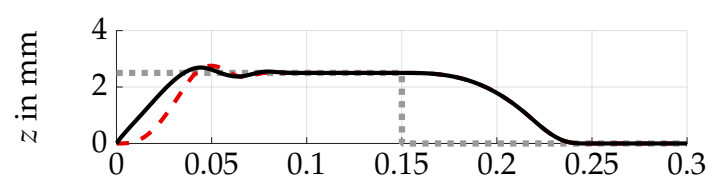

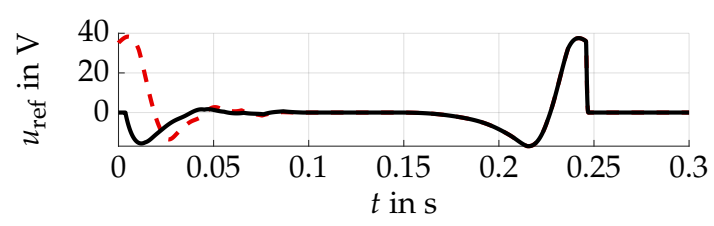

(a)

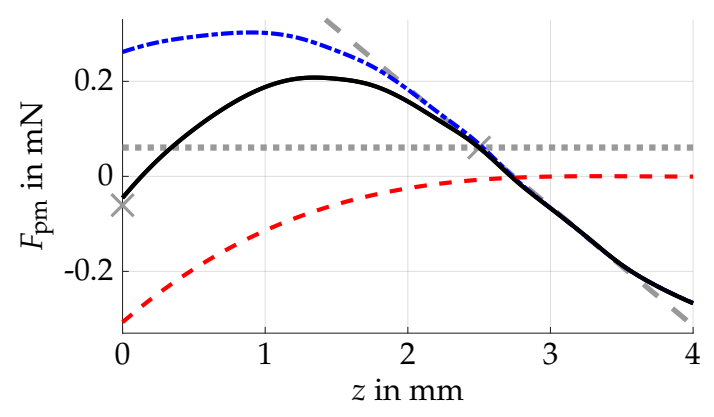

(b)

Figure 3. Optimised result: (a) The proof mass motion $z_{\text {ref }}(t)$ with corresponding input $u_{\text {ref }}(t)$, accelerated by a kick (solid, black), and an optimised trajectory without kick (dashed, red). The dotted, grey line shows the reference equilibrium over time. (b) The magnetic field (solid, black), resulting from the superposition of the two single magnetic fields (dashed, red and dashed-dotted, blue), nearly fulfils the target specifications (dashed and crosses, grey). The gravitational force is illustrated by the grey, dotted line.

simulation is different than that used for computing the feedforward term. The reference magnetic field and its variations can be seen in Figure $4 b$, and the resulting controlled motion is visualised in Figure 4 a.

The controller is still able to follow the reference trajectory, despite force deviations at the magnitude comparable to the gravitational force. The large motion deviation at the beginning is partly due to the fact that the kick has not been optimised for such forces pushing the movable mass against the piezo actuator. Thus, the significant increase in the solenoid voltage can be explained by the decreasing effect of the kick.

\section{Discussion}

The simulations illustrate the ability of the co-design algorithm to handle the contradictory goals effectively as well as finding a suitable cooperation strategy. The initial kick greatly reduces the load on the solenoid. However, high inputs are still needed for the transition to the lower equilibrium. Decreasing the distance between the solenoid and the lower equilibrium can lead to stability issues, since the proof mass cannot be controlled near the solenoid centre. This could be solved by using an additional electromagnet. The implemented feedback control strategy shows good results in terms of compensating significant differences in the magnetic fields. It can be seen, however, that the proof mass cannot be guaranteed to remain below the solenoid centre for larger disturbances, resulting in undesired input voltage spikes, as seen in Figure 4a for the green line. Similar to the high voltages for the downwards motion, this can be avoided by using a second solenoid. In further studies, the number of stable equilibria will be increased together with the working range by additional electroand permanent magnets. The simulative control approach currently assumes perfect state feedback. For the real system, however, a measurement system will be necessary. To this end, induction coils will be considered. Since only the velocity can be measured directly, an observer structure can reconstruct the needed signals. First approaches will be implemented in simulation and combined with the controller. Moreover, an optimal placement of both actuation and sensing coils will be a non-trivial task. Therefore, an extension of the presented co-design framework can be considered.

\section{Conclusions}

In this paper, we presented a novel, bistable microactuator design, which allows fast and free motion of a magnetic proof mass in vertical direction. For the transition between the equilibrium positions, a cooperative mechanism between a piezoelectric stack actuator and an electromagnetic solenoid is employed. We showed that the achievable efficiency in terms of input effort depends on 

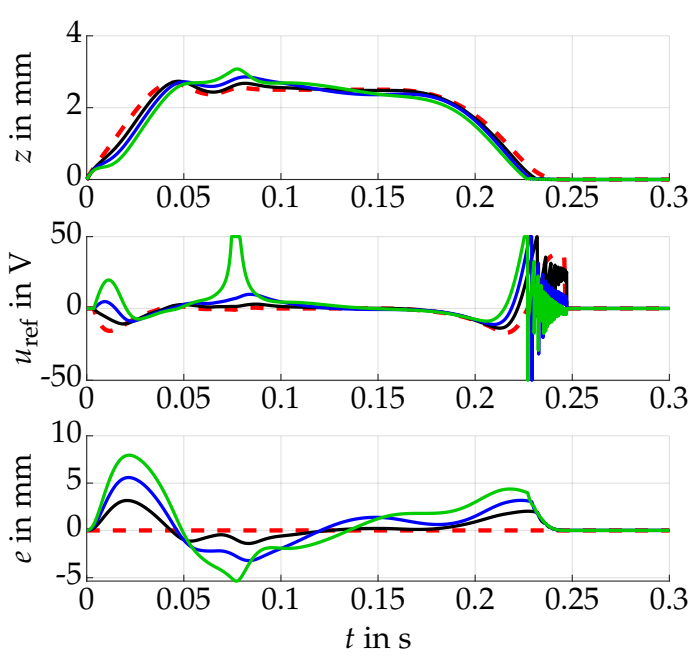

(a)

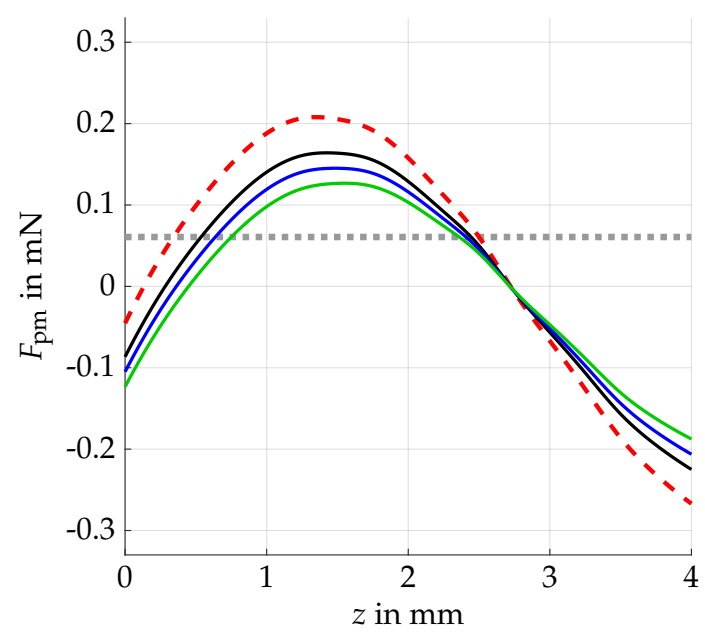

(b)

Figure 4. Flatness-based following control with model uncertainty is implemented. (a) The feedback controller is mostly able to compensate the model differences, but for the largest magnetic field deviation (solid, green), the position of the proof mass shortly coincides with the solenoid centre. The dashed, red line corresponds to the reference. (b) In each simulation, the magnetic force deviates more from the reference (dashed, red).

both, the design and the controller. A co-design approach has therefore successfully been applied to optimise the setup and trajectory at the same time, illustrating its usefulness for designing cooperative actuators. To follow a given reference trajectory, we designed a flatness-based controller and showed its effectiveness in terms of disturbance compensation in simulation. The efficiency and travel range of the overall setup can even be increased by an additional solenoid, since this cancels the restriction to remain below the solenoid centre.

Author Contributions: Conceptualization, C.A., U.W. and T.B.; Funding acquisition, C.A., U.W. and T.B.; Methodology, M.O.; Software, M.O.; Investigation, M.O., A.S. and K.K.; Writing-original draft preparation, M.O. and K.K.; Visualization, M.O.; Supervision, C.A., U.W. and T.B. All authors have read and agreed to the published version of the manuscript.

Funding: This research was funded by the German Research Foundation (DFG) within the priority program SPP2206 "KOMMMA", project "Kick and Catch - Cooperative Microactuators for Freely Moving Platforms"

Conflicts of Interest: The authors declare no conflict of interest.

\section{References}

1. Breguet, J.M.; Pérez, R.; Bergander, A.; Schmitt, C.; Clavel, R.; Bleuler, H. Piezoactuators for Motion Control from Centimeter to Nanometer. Proceedings of the IEEE/RSJ International Conference on Intelligent Robots and Systems, Takamatsu, Japan, 2000, pp. 492-497.

2. Mita, M.; Arai, M.; Tensaka, S.; Kobayashi, D. Fujita, H. A Micromachined Impact Microactuator Driven by Electrostatic Force. JMEMS 2003, 12, pp. 37-41, DOI: 10.1109/JMEMS.2002.802906.

3. Edeler, C.; Meyer, I.; Fatikow, S. Modeling of stick-slip micro-drives. J. Micro-Nano Mech. 2011, 6, pp. 65-87, DOI: $10.1007 /$ s12213-011-0034-9.

4. Ruffert, C.; Li, J.; Denkena, B.; Gatzen, H.H. Development and Evaluation of an Active Magnetic Guide for Microsystems With an Integrated Air Gap Measurement System. IEEE Trans. Magn. 2007, 43 pp. 2716-2718, DOI: 10.1109/TMAG.2007.893779.

5. Ruffert, C.; Gehrking, R.; Ponick, B.; Gatzen, H.H. Magnetic Levitation Assisted Guide for a Linear Micro-Actuator. IEEE Trans. Magn. 2006, 42 pp. 3785-3787, DOI: 10.1109/TMAG.2006.879160.

6. Poletkin, K.; Lu, Z.; Wallrabe, U.; Korvink, J.G.; Badilita, V. Stable dynamics of micro-machined inductive contactless suspensions. Int. J. Mech. Sci. 2017, 131 pp. 753-766, DOI: 10.1016/j.ijmecsci.2017.08.016. 
7. Safaeian, R.; Heydari, H. Optimal design of passive permanent magnet bearings. 27th Iranian Conference on Electrical Engineering, Yazd, Iran, 2019, pp. 458-463.

8. Tzes, A.; Nikolakopoulos, G.; Dritsas, L.; Koveos, Y. Multi-Parametric $H_{\text {inf }}$ Control of a Micro-actuator. $16 t h$ Triennial World Congress, Prague, Czech Republic, 2005, pp. 964-969.

9. Zhu, G.; Lévine, J.; Praly, L.; Peter, Y. Flatness-Based Control of Electrostatically Actuated MEMS With Application to Adaptive Optics: A Simulation Study. JMEMS 2006, 15 pp. 1165-1174, DOI: 10.1109/JMEMS.2006.880198.

10. Owusu, K.O.; Lewis, F.L. Solving the "Pull-in” Instability Problem of Electrostatic Microactuators using Nonlinear Control Techniques. Proceedings of the 2nd IEEE International Conference on Nano/Micro Engineered and Molecular Systems, Bangkok, 2007, pp. 1190-1195.

11. Zhu, G.; Saydy, L. Robust Output Feedback Control of an Electrostatic Micro-Actuator. Proceedings of the American Control Conference, New York, New York, 2007, pp. 3192-3197.

12. Peters, D.L.; Kurabayashi, K.; Papalambros, P.Y.; Ulsoy, A.G. Co-Design of a MEMS Actuator and Its Controller Using Frequency Constraints. ASME Dynamic Systems and Control Conference, Ann Arbor, Michigan, 2008, pp. 801-807.

13. Schütz, A.; Hu, S.; Rudnyi, E.B.; Bechtold, T. Electromagnetic System-Level Model of Novel Free Flight Microactuator. $21^{\text {st }}$ International Conference on Thermal, Mechanical and Multi-Physics Simulation and Experiments in Microelectronics and Microsystems, Cracow, Poland, 2020, pp. 1-6.

14. Goldfarb, M.; Celanovic, N. A lumped parameter electromechanical model for describing the nonlinear behavior of piezoelectric actuators. J. Dyn. Sys., Meas., Control 1997, 119, pp. 478-485, DOI: 10.1115/1.2801282.

15. Main, J.A.; Garcia, E. Piezoelectric Stack Actuators and Control System Design: Strategies and Pitfalls. J. Guid. Control Dynam. 1997, 20, pp. 478-485, DOI: 10.2514/2.4066.

16. Agashe, J.s.; Arnold, D.P. A study of scaling and geometry effects on the forces between cuboidal and cylindrical magnets using analytical force solutions. J. Phys. D: Applied Physics 2008, 41, pp. 1-9, DOI: 10.1088/0022-3727/42/9/099801.

17. Ravaud, R.; Lemarquand, G.; Babic, S.; Lemarquand, V.; Akyel, C. Cylindrical Magnets and Coils: Fields, Forces, and Inductances. IEEE Trans. Magn. 2010, 46, pp. 3585-3590, DOI: 10.1109/TMAG.2010.2049026.

18. Ansys Inc. ANSYS Electronics Desktop, Release 2020 R1, 2020.

19. Specker, T.; Buchholz, M.; Dietmayer, K. Dynamical Modeling of Constraints with Friction in Mechanical Systems. 8th Vienna International Conference on Mathematical Modelling, Vienna, Austria, 2015, pp. 514-519.

20. Fliess, M.; Lévine, J.; Martin, P.; Rouchon, P. Flatness and defect of non-linear systems: introductory theory and examples. Int. J. Control 1995, 61, pp. 1327-1361, DOI: 0.1080/00207179508921959.

21. Charlet, B.; Lévine, J. On dynamic feedback linearization. Syst. Control Lett. 1989, 13, pp. 143-151, DOI: 10.1016/0167-6911(89)90031-5.

22. The MathWorks Inc. MATLAB and Global Optimization Toolbox (R2019a) Natick, Massachusetts, 2019. 